\title{
Outcomes of Cardiopulmonary Resuscitation in the Pediatric Intensive Care of a Tertiary Center
}

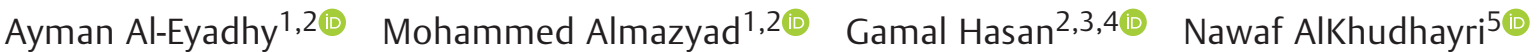 \\ Abdullah F. AlSaeed ${ }^{5(1)}$ Mohammed Habib $^{5(-)}$ Ali A. N. Alhaboob ${ }^{1,2(0)}$ Mohammed AlAyed $^{5(0)}$ \\ Yazeed AlSehibani ${ }^{5}$ Fahad Alsohime ${ }^{1,2}$ Majed Alabdulhafid ${ }^{1,2}$ Mohamad-Hani Temsah ${ }^{1,2}$
}

${ }^{1}$ Department of Pediatrics, College of Medicine, King Saud University, Riyadh, Saudi Arabia

2 Department of Pediatrics, Pediatric Intensive Care Unit, King Saud

Address for correspondence Ayman A. Al-Eyadhy, MD, Department of Pediatrics, College of Medicine, Pediatric Intensive Care Unit, King University Medical City, King Saud University, Riyadh, Saudi Arabia Saud University, P.O. Box 2925, Riyadh 11461, Saudi Arabia

${ }^{3}$ Department of Pediatrics, Assiut Faculty of Medicine, Assiut University, Assiut, Egypt

${ }^{4}$ Department of Pediatrics, Pediatric Critical Care Unit, Sheikh Shakhbout Medical City, Abu Dhabi, United Arab Emirates

${ }^{5}$ College of Medicine, King Saud University, Riyadh, Saudi Arabia (e-mail: aleyadhy@ksu.edu.sa).

J Pediatr Intensive Care

\begin{abstract}
Keywords

- cardiopulmonary resuscitation

- pediatric

- survival

- pediatric intensive care unit

- Saudi

Understanding the factors affecting survival and modifying the preventable factors may improve patient outcomes following cardiopulmonary resuscitation (CPR). The aim of this study was to assess the prevalence and outcomes of cardiac arrest and CPR events in a tertiary pediatric intensive care unit (PICU). Outcomes of interest were the return of spontaneous circulation (ROSC) lasting more than 20 minutes, survival for 24 hours postCPR, and survival to hospital discharge. We analyzed data from the PICU CPR registry from January 1, 2011 to January 1, 2018. All patients who underwent at least 2 minutes of CPR in the PICU were included. CPR was administered in 65 PICU instances, with a prevalence of $1.85 \%$. The mean patient age was 32.7 months. ROSC occurred in 38 (58.5\%) patients, 30 (46.2\%) achieved 24-hour survival, and 21 (32.3\%) survived to hospital discharge. Younger age $(p<0.018)$, respiratory cause $(p<0.001)$, bradycardia $(p<0.018)$, and short duration of CPR $(p<0.001)$ were associated with better outcomes, while sodium bicarbonate, norepinephrine, and vasopressin were associated with worse outcome $(p<0.009)$. The offhour CPR had no impact on the outcome. The patients' cumulative predicted survival declined by an average of $8.7 \%$ for an additional 1 minute duration of CPR $(p=0.001)$. The study concludes that the duration of CPR, therefore, remains one of the crucial factors determining CPR outcomes and needs to be considered in parallel with the guideline emphasis on CPR quality. The lower survival rate post-ROSC needs careful consideration during parental counseling. Better anticipation and prevention of CPR remain ongoing challenges.
\end{abstract}

received

May 4, 2021

accepted after revision

July 4, 2021
DOI https://doi.org/

10.1055/s-0041-1733855. ISSN 2146-4618. (c) 2021. The Author(s).

This is an open access article published by Thieme under the terms of the Creative Commons Attribution-NonDerivative-NonCommercial-License, permitting copying and reproduction so long as the original work is given appropriate credit. Contents may not be used for commercial purposes, or adapted, remixed, transformed or built upon. (https://creativecommons.org/ licenses/by-nc-nd/4.0/)

Georg Thieme Verlag KG, Rüdigerstraße 14, 70469 Stuttgart, Germany 


\section{Introduction}

The prevalence of cardiopulmonary resuscitation (CPR) in hospitalized children in pediatric wards and pediatric intensive care units (PICU) varies from 2 to $6 .^{1}$ Alten et al reported a cardiac arrest prevalence of 3.1\% among 15,908 cardiac PICU encounters. ${ }^{2}$ Over the past decade, a significant increase in CPR events has occurred in the PICU compared with the wards. ${ }^{3}$ Nevertheless, pediatric resuscitation represents an underappreciated subject, as reported by a recent systematic analysis. ${ }^{4}$ Moreover, the outcomes of cardiac arrest in pediatric patients depend on multiple factors, including the quality of the CPR and postresuscitation care. Improvements in these factors are associated with improvements in the rates of return of spontaneous circulation (ROSC) by up to $75 \%{ }^{5,6}$

Survival to hospital discharge is an area of intense interest in the resuscitation literature. For example, Topjian et al, in their systematic review, reported a wide range of 25 to $50 \%$ survival and discharge from hospital in pediatric patients after CPR. ${ }^{7}$ Higher rates of CPR survival to discharge have also been reported in pediatric cardiac ICUs. One report from a cardiac center showed a rate of survival to discharge as high as $50 \%{ }^{8}$ A collective review of pediatric CPR cases reported better outcomes for respiratory cases, with $75 \%$ of these patients surviving to discharge compared with only $13 \%$ of cardiac cases. ${ }^{9,10}$

CPR duration is one key factor affecting the CPR outcome. The longer the CPR is performed, the less chance the patient has for survival. For instance, a CPR duration of more than 15 to 20 minutes was predicted to yield a poor outcome, while a CPR duration of less than 10 minutes was predicted to yield a better outcome. ${ }^{11,12}$ However, performing CPR for more than 20 minutes is not always futile. Thus, other key factors, such as CPR quality and postresuscitation care, are of similar importance. Matos et al illustrated that $12 \%$ of patients undergoing CPR for more than 35 minutes survived. ${ }^{12}$ Therefore, continuing CPR after the 20 minutes mark may yield a favorable neurological outcome for patients who would have died had CPR not been continued. ${ }^{12}$ All these studies indicate that the duration of CPR is an important consideration. However, CPR duration is not independent of other factors that also affect outcomes, such as the CPR quality and the setting of the CPR events.

The observed differences in survival rates suggest that the outcome of CPR is a moving target, with various factors that require further exploration. In this study, we assessed the prevalence and outcomes of CPR in the PICU and explored the factors affecting CPR outcomes, including sex, age, causes of cardiac arrest, diagnoses, cardiac arrest rhythm, working hours, duration of the CPR time, and medications.

\section{Materials and Methods}

\section{Patients and Data Collection}

This retrospective cohort study was conducted in the PICU of King Khalid University Hospital. Inclusion criteria were patients ranging in age from 0 to 14 years old who were admitted to the PICU, underwent CPR after PICU admission, and had CPR for at least 2 minutes. ${ }^{13}$ Patients with incomplete data were excluded. We included all consecutive patients who met the inclusion criteria during the study period from January 1, 2011 to January 1, 2018. Outcomes of interest were a return of spontaneous circulation (ROSC) lasting more than 20 minutes, survival for 24-hour post-CPR, and survival to hospital discharge.

The data were retrospectively collected from the PICU CPR registry and electronic medical records. The collected data included demographic variables such as age, weight, and sex, and the clinical variables such as type of arrest, diagnosis, first documented rhythm, duration of CPR, time of arrest, and medications used during CPR. The working hours were defined as 07:00 A.M. to 16:00 P.M. during weekdays, while the remaining hours were considered on-call hours.

\section{Statistical Analysis}

SPSS IBM v24 was used to statistically analyze the data. Means and standard deviations were used to describe continuous variables such as age and weight, while frequencies and percentages were employed to describe categorical variables such as sex and treatments. The Chi-square test was used to assess the association between categorical variables. The independent samples $t$-test was used to assess statistical differences in continuous variables across the patients' dichotomous survival levels. The Cochran's Q Chisquare test was also used to assess the association between children's repeated survival measures over time (immediate, at 24 hours, and at discharge).

Because of the repeated survival measures and the declining survival of children in the PICU after cardiopulmonary arrest over time, we analyzed the repeated binary survival measures using the generalized estimating equation (GEE) logistic regression analysis. The GEE logistic regression analysis is a repeated measures analysis of survival over time. We employed the GEE quasi-likelihood estimation method as a default. However, the model was further evaluated for goodness of fit by using the quasi-information criterion and Akaike's information criterion compared with an empty model with no predictors. The association between the analyzed predictors of children's survival over time was expressed as an exponential distribution $(\beta)$. Figures were created by using SPSS and Excel programs. The $\alpha$ significance level was set to 0.05 throughout the analysis.

\section{Ethical Considerations}

The study was initiated upon receiving approval from the institutional review board (IRB) of the College of Medicine at King Saud University, Kingdom of Saudi Arabia. Confidentiality of patient identifications was maintained by converting the medical record numbers to coded numbers with no identifiers. Therefore, patient consent was waived by the IRB.

\section{Results}

- Table 1 summarizes the demographic and other characteristics of the study cohort. A total of 70 cases that fit the inclusion criteria were found in the PICU CPR registry. We 
Table 1 Demographics and characteristics of children admitted to the pediatric intensive care unit and requiring cardiopulmonary resuscitation

\begin{tabular}{|c|c|c|}
\hline Variable & Frequency & Percentage \\
\hline \multicolumn{3}{|l|}{ Sex } \\
\hline Male & 37 & 56.9 \\
\hline Female & 28 & 43.1 \\
\hline \multicolumn{3}{|l|}{ Age (mo) } \\
\hline Mean $( \pm S D)$ & \multicolumn{2}{|l|}{$32.7(51.1)$} \\
\hline Median (Q25-Q75) & \multicolumn{2}{|l|}{$6(3-35)$} \\
\hline$<6$ mo & 33 & 50.8 \\
\hline$>6-12 \mathrm{mo}$ & 7 & 10.8 \\
\hline $13-48 \mathrm{mo}$ & 11 & 16.9 \\
\hline$>48 \mathrm{mo}$ & 14 & 21.5 \\
\hline Weight $(\mathrm{kg})$, mean $( \pm \mathrm{SD})$ & \multicolumn{2}{|c|}{$11.10( \pm 13.7)$} \\
\hline \multicolumn{3}{|l|}{ Causes of cardiac arrest } \\
\hline Cardiac & 26 & 40 \\
\hline Respiratory & 39 & 60 \\
\hline \multicolumn{3}{|l|}{ Diagnoses } \\
\hline CVS & 5 & 7.7 \\
\hline Respiratory & 23 & 35.4 \\
\hline Sepsis & 14 & 21.5 \\
\hline Others & 23 & 35.4 \\
\hline $\begin{array}{l}\text { Pre-arrest endotracheal } \\
\text { tube }\end{array}$ & 55 & 84.6 \\
\hline $\begin{array}{l}\text { Pre-arrest noninvasive } \\
\text { ventilation }\end{array}$ & 2 & 3.1 \\
\hline \multicolumn{3}{|l|}{ Time of arrest } \\
\hline Working hours & 31 & 47.7 \\
\hline On-call hours & 34 & 52.3 \\
\hline
\end{tabular}

Abbreviations: CVS, cardiovascular system; SD, standard deviation.

excluded five cases that had missing data. Thus, a total of 65 cases were included in the analysis. Although the mean patient age was $32.7 \pm 51.1$ months, 40 (61.6\%) patients were younger than 12 months. The study cohort consisted of 37 (56.9\%) males and 23 (43.1\%) females. Most of the presenting illnesses were respiratory (23 cases, $35.4 \%$ ), and a respiratory cause of arrest was attributed to $39(60 \%)$ cases. Furthermore, $55(84.6 \%)$ patients were intubated and mechanically ventilated before the cardiopulmonary arrest. The time of arrest was almost equally distributed, with 34 (52.3\%) and 31 (47.7\%) arrests occurring during working hours and on-call hours, respectively.

The CPR details, medications used, and outcomes are shown in -Table 2. Bradycardia was the most common cardiac rhythm during the arrest, occurring in 58 (89.2\%) cases. The mean duration of CPR was $18.34 \pm 15.9$ minutes. While 38 (58.5\%) patients achieved ROSC post-CPR, only 30 (46.2\%) patients survived for more than 24 hours, and only 21 (32.3\%) patients survived to discharge, as shown in - Fig. 1.
Table 2 Cardiopulmonary resuscitation events, medications, and outcomes of children who underwent cardiopulmonary resuscitation in the pediatric intensive care unit

\begin{tabular}{|c|c|c|}
\hline Variables & Frequency & Percentage \\
\hline \multicolumn{3}{|l|}{ Cardiac arrest rhythm } \\
\hline Sinus bradycardia & 58 & 89.2 \\
\hline $\begin{array}{l}\text { Shockable } \\
\text { tachyarrhythmia }\end{array}$ & 3 & 4.6 \\
\hline $\begin{array}{l}\text { Nonshockable } \\
\text { dysrhythmias }\end{array}$ & 4 & 6.2 \\
\hline \multicolumn{3}{|l|}{ Duration of the CPR time (min) } \\
\hline Mean $( \pm S D)$ & \multicolumn{2}{|l|}{$18.3(15.9)$} \\
\hline Median (IQR) & \multicolumn{2}{|l|}{$12(61)$} \\
\hline $\begin{array}{l}\text { Immediate CPR outcome; } \\
\text { ROSC }\end{array}$ & 38 & 58.5 \\
\hline $\begin{array}{l}\text { First } 24 \text { h outcome; } \\
\text { survived }\end{array}$ & 30 & 46.2 \\
\hline $\begin{array}{l}\text { Discharge final outcome; } \\
\text { survived }\end{array}$ & 21 & 32.3 \\
\hline \multicolumn{3}{|l|}{ Medication (IV bolus) } \\
\hline Epinephrine & 59 & 90.8 \\
\hline Sodium bicarbonate & 21 & 32.3 \\
\hline Atropine & 17 & 26.2 \\
\hline Normal saline & 16 & 24.6 \\
\hline Calcium & 12 & 18.5 \\
\hline Lidocaine & 3 & 4.6 \\
\hline Adenosine & 2 & 3.1 \\
\hline Albumin 5\% & 2 & 3.1 \\
\hline Amiodarone & 1 & 1.5 \\
\hline Magnesium sulfate & 1 & 1.5 \\
\hline \multicolumn{3}{|l|}{ Medication (IV infusion) } \\
\hline Epinephrine & 38 & 58.5 \\
\hline Norepinephrine & 20 & 30.8 \\
\hline Dopamine & 18 & 27.7 \\
\hline Dobutamine & 13 & 20 \\
\hline Vasopressin & 6 & 9.2 \\
\hline Phenylephrine & 6 & 9.2 \\
\hline Amiodarone & 3 & 4.6 \\
\hline Sodium bicarbonate & 1 & 1.5 \\
\hline
\end{tabular}

Abbreviations: CPR, cardiopulmonary resuscitation; IQR, interquartile range; ROSC, return of spontaneous circulation; SD, standard deviation.

The bivariate analysis and comparison between patients with and without ROSC after CPR revealed that a younger age was significantly associated with a better ROSC outcome after CPR. By contrast, deceased children were significantly older (51.8 \pm 60.9 months) than children who survived (19.1 \pm 38.1 months) and successfully achieved ROSC $(p=0.018)$. For further analysis, the patients were classified into different age 


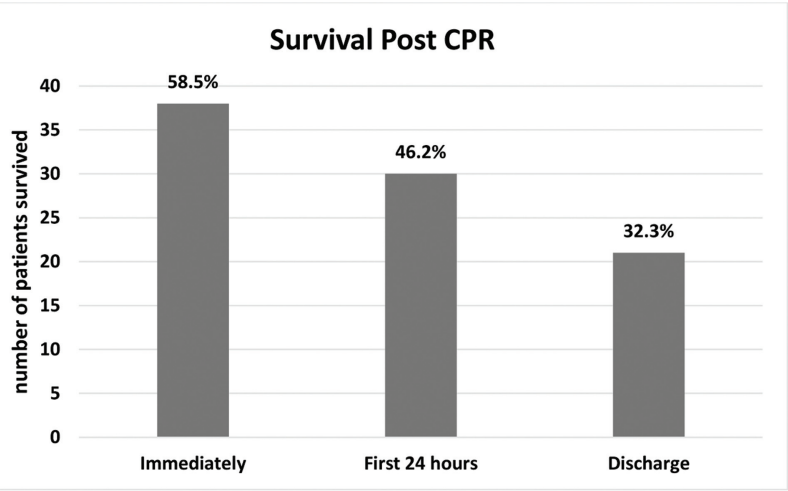

Fig. 1 Survival after CPR, including immediate return of spontaneous circulation, survival for the first 24 hours after CPR, and survival at time of discharge $($ Chi-square $=25.5, p$-value $<0.001)$.

intervals to account for outliers. This classification revealed that children older than 48 months were predicted to have worse outcomes after CPR than were children within younger age intervals $(p=0.015)$.

- Table 3 shows the detailed bivariate analysis and comparisons between patients with and without ROSC after CPR. ROSC outcomes were better for patients with a respiratory cause than with a cardiac cause of arrest; 30 (78.9\%) patients who survived had a respiratory cause of the arrest $(p<0.001)$. By contrast, those with sepsis were marginally less likely to survive CPR than children with cardiovascular diseases, respiratory diseases, and other diseases. The CPR duration was longer for deceased patients ( $31.2 \pm 15$ minutes) than for patients who reached ROSC ( $9.2 \pm 8.5$ minutes). Treatments with a sodium bicarbonate bolus or with norepinephrine or vasopressin infusions during CPR were significantly associated with higher mortality and less ROSC.

The GEE logistic regression analysis was conducted by setting the patients as subjects and the time as a repeated measure, after transposing the data into the long format yielding $65 \times 3=195$ patient records. The analysis revealed that bradycardia, duration of CPR, and absence of norepinephrine infusion were significantly associated with higher accumulative survival until discharge ( - Table 4 ). Moreover, the analysis model showed that CPR duration predicted significantly shorter survival: an increase in the CPR duration of 1 minute decreased the children's cumulative predicted survival by an average of $8.7 \%(p=0.001)$. The GEE analysis also showed that an increase in the CPR duration from 3 to 6 minutes resulted in a nearly steady predicted survival; however, survival declined for CPR durations of 7 to 9 minutes and plunged more rapidly for CPR durations of 10 minutes or more. This negative association between CPR duration and survival was statistically significant when considering the other factors in the model (-Fig. 2).

\section{Discussion}

This retrospective study involved PICU patients and included 65 documented instances of at least 2 minutes of CPR. The purpose of the study was to assess the frequency, survival, and variables affecting CPR outcomes at a PICU. In our study, 38 (58.5\%) patients achieved ROSC, which is consistent with two previous studies. ${ }^{14,15}$ Our examination of 24-hour survival also revealed that 30 (46.2\%) patients reached 24 -hour survival. This value was similar to the $58.4 \% 24$-hour survival rate reported in a multicenter study investigating survival trends on weekends compared with weekdays, but it was slightly lower than the 64\% survival in PICU patients 24 hours after CPR reported in another multicenter study conducted in the United States. ${ }^{12,13}$ The contrasting results between the survivals to discharge in these studies may be due to multiple factors. One explanation might be the lack of reporting data related to CPR quality and postcardiac arrest care. Differences in the inclusion criteria could also contribute to these varying outcomes. For instance, we excluded patients who had undergone CPR for less than 2 minutes, and this exclusion might have lowered the survival to discharge reported by us and by Bhanji et al, ${ }^{13}$ compared the rate reported by Matos et al. ${ }^{12}$

In our study, 21 (32.3\%) patients survived to discharge. This rate was slightly below the rates reported in three previous studies, which showed survival to discharge rates of 45, 36.2, and $34.8 \%$ after CPR in PICUs. ${ }^{1,11,13}$ Variations in survival rates to discharge are expected, as differences in diagnoses, severity, comorbidity, and age can influence the survival to discharge rates. Once again, the differences in inclusion criteria and the lack of consistent reporting of CPR quality could contribute to the discrepancy in results seen in the reported survival rates. ${ }^{16-18}$ High-quality CPR is the first take-home message of the American Heart Association (AHA) in their 2020 Pediatric Basic and Advanced Life Support update. ${ }^{19}$ The literature confirms that the key components of high-quality CPR are applying adequate chest compression rate and depth, minimizing CPR interruptions, allowing full chest recoil between compressions, and preventing excessive ventilation. ${ }^{19}$

We noticed that ROSC was higher among our patients who were less than 6 months of age, as 22 (57.9\%) patients under 6 months of age achieved ROSC versus 16 (42.1\%) patients older than 6 months. This is consistent with a significantly higher survival rate in infants and neonates (53.2 and 62\%, respectively) compared with older children (29.8-41.2\%) reported in a recent study conducted in the United States. ${ }^{20}$ Another recently published study on CPR epidemiology in PICUs across England revealed similar results. ${ }^{21}$ By contrast, worse outcomes were reported for infants than for older children following out-of-hospital CPR. ${ }^{22-24}$ Infants have less respiratory reserve and are therefore likely to have a respiratory cause for CPR. Thus, any delay in recognizing or providing respiratory support during out-of-hospital CPR can be detrimental, whereas patients in the PICU are carefully monitored and are less likely to have a delay in respiratory support. Therefore, lower survival rates were correlated with the advancing age of patients who underwent CPR in the PICU setting compared with out-of-hospital CPR.

Our study showed that outcomes were significantly better when the cause of arrest was respiratory related. A majority of patients (78.9\%) who underwent CPR and survived had a respiratory cause of arrest. This is compatible with results from another study showing a better survival outcome for 
CPR Outcomes in the PICU Al-Eyadhy et al.

Table 3 Bivariate analysis of clinical characteristics associated with an immediate revival after cardiopulmonary resuscitation in the pediatric intensive care unit

\begin{tabular}{|c|c|c|c|c|}
\hline & \multicolumn{2}{|c|}{ (Outcome) ROSC } & \multirow[b]{2}{*}{ Test statistic } & \multirow[b]{2}{*}{$p$-Value } \\
\hline & $\begin{array}{l}\text { Deceased } \\
n=27\end{array}$ & $\begin{array}{l}\text { Revived } \\
n=38\end{array}$ & & \\
\hline \multicolumn{5}{|l|}{ Sex } \\
\hline Male & $15(55.6 \%)$ & $22(57.9 \%)$ & $X^{2}(1)=0.04$ & 0.851 \\
\hline Female & $12(44.4 \%)$ & $16(42.1 \%)$ & & \\
\hline Age $(\mathrm{mo})$, mean $( \pm \mathrm{SD})$ & $51.8(60.9)$ & $19.1(38.1)$ & $t(40.3)=2.5$ & 0.018 \\
\hline$\leq 6 \mathrm{mo}$ & $11(40.7 \%)$ & $22(57.9 \%)$ & $X^{2}(3)=10.41^{*}$ & 0.015 \\
\hline$>6-12 \mathrm{mo}$ & $2(7.4 \%)$ & $5(13.2 \%)$ & & \\
\hline $13-48 \mathrm{mo}$ & $3(11.1 \%)$ & $8(21.1 \%)$ & & \\
\hline$>48$ Months & $11(40.7 \%)$ & $3(7.9 \%)$ & & \\
\hline Weight $(\mathrm{kg})$, mean $( \pm \mathrm{SD})$ & $16.5(19)$ & $7.3(6.5)$ & $t(28.9)=2.40$ & 0.025 \\
\hline \multicolumn{5}{|l|}{ Causes of cardiac arrest } \\
\hline Cardiac & $18(66.7 \%)$ & $8(21.1 \%)$ & $X^{2}(1)=13.7^{*}$ & $<0.001$ \\
\hline Respiratory & $9(33.3 \%)$ & $30(78.9 \%)$ & & \\
\hline \multicolumn{5}{|l|}{ Diagnoses } \\
\hline CVS & $2(7.4 \%)$ & $3(7.9 \%)$ & $x^{2}(3)=7.60^{*}$ & 0.056 \\
\hline Respiratory & $6(22.2 \%)$ & $17(44.7 \%)$ & & \\
\hline Sepsis & $10(37 \%)$ & $4(10.5 \%)$ & & \\
\hline Others & $9(33.3 \%)$ & $14(36.8 \%)$ & & \\
\hline \multicolumn{5}{|l|}{ Cardiac arrest rhythm } \\
\hline Sinus bradycardia & $22(81.5 \%)$ & $36(94.7 \%)$ & $X^{2}(2)=2.93^{*}$ & 0.231 \\
\hline Shockable tachyarrhythmia & $2(7.4 \%)$ & $1(2.6 \%)$ & & \\
\hline Nonshockable dysrhythmias & $3(11.1 \%)$ & $1(2.6 \%)$ & & \\
\hline Pre-arrest intubation & $26(96.3 \%)$ & $29(76.3 \%)$ & $x^{2}(1)=3.43^{* *}$ & 0.064 \\
\hline Pre-arrest noninvasive ventilation & $1(3.7 \%)$ & $1(2.6 \%)$ & $x^{2}(1)<0.001^{* *}$ & $1=\mathrm{NS}$ \\
\hline \multicolumn{5}{|l|}{ Time of arrest } \\
\hline Working hours & $10(3 \%)$ & $21(55.3 \%)$ & $x^{2}(1)=2.10$ & 0.147 \\
\hline On-call hours & $17(63 \%)$ & $17(44 \%)$ & & \\
\hline Duration of the CPR time (min) & $31.2(15)$ & $9.2(8.5)$ & $t(37.9)=6.9$ & $<0.001$ \\
\hline \multicolumn{5}{|l|}{ Medications } \\
\hline Epinephrine IV bolus & $25(92.6 \%)$ & 34 (89.5\%) & $x^{2}(1)<0.001^{* *}$ & NS \\
\hline Lidocaine IV bolus & $1(3.7 \%)$ & $2(5.3 \%)$ & $\mathrm{x}^{2}(1)<0.001^{* *}$ & NS \\
\hline Sodium bicarbonate IV bolus & 14 (51.9\%) & $7(18.4 \%)$ & $x^{2}(1)=8.1$ & 0.005 \\
\hline Calcium chloride/gluconate IV bolus & $8(29.6 \%)$ & $4(10.5 \%)$ & $x^{2}(1)=2.70$ & 0.103 \\
\hline Bolus amiodarone & $1(3.7 \%)$ & 0 & $x^{2}(1)=0.030^{* *}$ & 0.862 \\
\hline Atropine IV bolus & $7(25.9 \%)$ & $10(26.3 \%)$ & $x^{2}(1)<0.001^{* *}$ & NS \\
\hline Adenosine bolus & $1(3.7 \%)$ & $1(2.6 \%)$ & $x^{2}(1)<0.001$ & NS \\
\hline Magnesium sulfate IV bolus & $1(3.7 \%)$ & 0 & $x^{2}(1)=0.030$ & 0.863 \\
\hline Norepinephrine infusion & $15(55.6 \%)$ & $5(13.2 \%)$ & $x^{2}(1)=13.3$ & $<0.000$ \\
\hline Dopamine infusion & $10(37 \%)$ & $8(21.1 \%)$ & $x^{2}(1)=2.01$ & 0.156 \\
\hline Vasopressin infusion & $6(22.2 \%)$ & 0 & $x^{2}(1)=6.004^{* *}$ & 0.009 \\
\hline
\end{tabular}

Abbreviations: CVS, cardiovascular system; CPR, cardiopulmonary resuscitation; ROSC, return of spontaneous circulation; SD, standard deviation. *Likelihood ratio adjusted Chi squared test.

${ }^{* *}$ Chi squared test with Yates correction. 


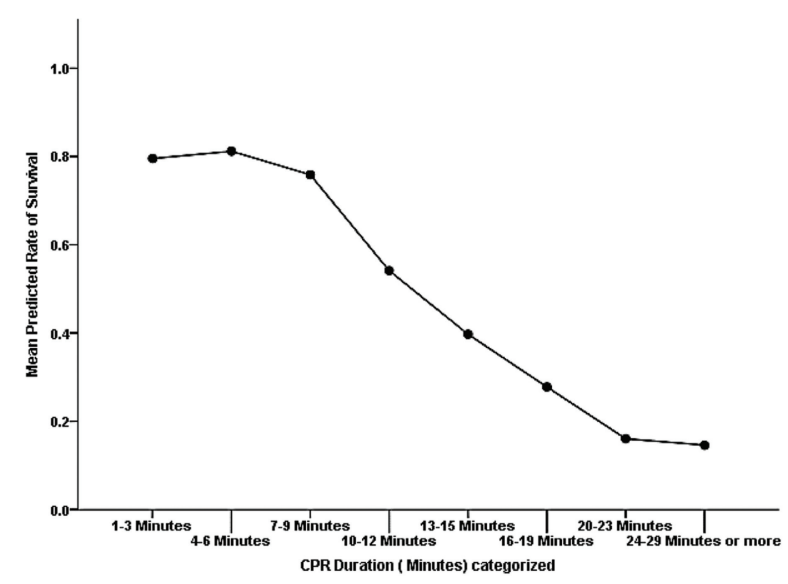

Fig. 2 The association between CPR duration (minutes) and the generalized estimating equation model predicted survival rate (probability) over time. CPR, cardiopulmonary resuscitation.

pediatric patients with respiratory arrest (64.9\%) than with cardiac-related arrest. ${ }^{25}$ The better outcomes in patients with respiratory-related arrest may be due to a shorter CPR duration or a better tolerance for hypoxia compared with a cessation of perfusion. Moreover, better survival may result from airway maneuvers that enhance oxygenation by recruiting functional alveolar segments. ${ }^{25}$

The use of medication during CPR was an important aspect of our study. Our aim was to identify the most frequently used medications and their effects on CPR outcomes. Only vasopressin, sodium bicarbonate, and norepinephrine were significantly associated with inferior outcomes, similar to the findings of other studies. ${ }^{15,26}$ This was partially due to the use of these medications as a last attempt at saving a patient, as well as the ambiguous indications for these medications during CPR. However, the use of epinephrine and atropine boluses was reported in an earlier study to improve the survival rate of patients. ${ }^{9}$

Our results demonstrated a mean CPR duration of 9.2 minutes for patients who achieved ROSC, while the mean CPR duration for patients that did not survive was 31.2 minutes. A previous study showed that the first 15 minutes of CPR had the best outcomes, with a $41 \%$ probability of survival. Alternatively, prolonged CPR that extended beyond 35 minutes had only a $12 \%$ probability of survival, indicating that survival decreased by $2.1 \%$ per 1 minute of CPR. ${ }^{12}$ Thus, CPR duration is inversely related to favorable outcomes. Our study showed similar findings, as the CPR duration was independently associated with survival to discharge.

The GEE model suggests a downward trend in ROSC after a CPR duration of 7 to 9 minutes, which may reflect prolonged oxygen deprivation and low perfusion during the arrest. Another explanation for the inverse relationship between CPR duration and survival is the waning effect of epinephrine after three to five doses, as some preclinical models have suggested. ${ }^{27}$ Furthermore, in contrast to adult and pediatric out-of-hospital studies, the time until defibrillation, when a shockable rhythm was detected, did not correlate with outcomes in the setting of in-hospital cardiac arrest. ${ }^{28} \mathrm{CPR}$ performed in a perioperative setting shares similar CPR contexts with the PICU setting. ${ }^{29}$

The diagnosis of pediatric patients also had a significant impact on the outcome of CPR. In our research, favorable CPR outcomes were significantly associated with respiratory diagnoses, as 17 (73.9\%) patients with a respiratory diagnosis on PICU admission achieved ROSC. Cardiac diagnoses are usually due to congenital diseases and are more chronic in nature than respiratory diagnoses, which are commonly associated with infectious causes. Furthermore, our sample size for cardiac diagnosis was small, as only five (7.69\%) patients had a cardiac diagnosis. Other studies have

Table 4 Generalized estimating equation model explaining repeated survival rate from immediate post-CPR until discharge time for pediatric intensive care unit patients who underwent CPR $(n=195)$

\begin{tabular}{|l|l|l|l|l|l|l|}
\hline Parameter & B & Standard error & Adjusted odds ratio & \multicolumn{2}{l|}{$95 \% \mathrm{Cl}$} & \multirow{2}{*}{$p$-Value } \\
\cline { 5 - 7 } & & & & Lower & Upper & \\
\hline (Intercept) & -2.635 & 0.8718 & 0.072 & 0.013 & 0.396 & 0.003 \\
\hline Female (sex) & 1.161 & 0.6213 & 3.192 & 0.945 & 10.788 & 0.062 \\
\hline Age (mo) & 0.004 & 0.0065 & 1.004 & 0.991 & 1.017 & 0.552 \\
\hline Cause of arrest & -0.697 & 0.5135 & 0.498 & 0.182 & 1.363 & 0.175 \\
\hline Bradycardia rhythm & 1.618 & 0.6859 & 5.045 & 1.315 & 19.353 & 0.018 \\
\hline Shockable tachycardia & -0.756 & 0.8912 & 0.470 & 0.082 & 2.693 & 0.396 \\
\hline Time working arrest & 0.228 & 0.5591 & 1.256 & 0.420 & 3.758 & 0.684 \\
\hline Duration of CPR & -0.091 & 0.0282 & $0.913^{\text {a }}$ & 0.864 & 0.965 & 0.001 \\
\hline No NaHCO3 bolus & -0.367 & 0.6318 & 0.693 & 0.201 & 2.390 & 0.561 \\
\hline No norepinephrine infusion & 2.955 & 0.5162 & 19.211 & 6.986 & 52.833 & $<0.001$ \\
\hline
\end{tabular}

Abbreviations: $\mathrm{Cl}$, confidence interval; CPR, cardiopulmonary resuscitation; ROSC, return of spontaneous circulation; SD, standard deviation. ${ }^{\mathrm{a}}(0.913$ times less accumulated survival $=(1-0.913) \times 100=8.7 \%$ less; as the CPR duration increased by 1 minute, the children's cumulative predicted survival declined by an average of $8.7 \%$. 
demonstrated better survival outcomes after CPR for pediatric patients with cardiac diagnoses. The difference between our study and others may reflect the larger sample sizes and higher numbers of cardiac arrest cases with cardiac diagnoses in the other studies. ${ }^{9,30}$

A recent study compared CPR survival rates after pediatric cardiac arrest during nights and weekends to CPR survival during day shifts and weekdays in a large database that included 12,404 children who received CPR in 354 hospitals. The survival rate for pediatric patients undergoing CPR was lower during the night shifts than in the day shifts, although no clear difference was noted between weekends and weekdays. ${ }^{13}$ Other recent studies have also demonstrated a marked drop-off in immediate ROSC and survival to discharge in patients who underwent CPR on weekends and during night shifts compared with weekdays and day shifts. ${ }^{31,32}$ However, our research found no statistically significant differences between patients who underwent CPR during regular working hours and patients who underwent CPR during on-call hours. The differences in our study and others may be due to each hospital's staff management, which includes adequate staff numbers during both shifts, and the 24-hour in-house presence of a senior PICU team member. However, the decline in CPR quality during the night, which is attributed to fatigue, has been an area of concern. Although reports evaluating children's blood pressure during chest compression suggested a decline in CPR quality at night, these findings were not consistent across reports. ${ }^{33-35}$

We found that the presence of an endotracheal tube and mechanical ventilation pre-arrest were marginally correlated with a lower chance of survival, in line with other published papers. ${ }^{12,31,36}$ Patients who develop cardiac arrest despite receiving respiratory assistance may have a worse prognosis due to their underlying disease severity. Moreover, the concept of reperfusion injury due to free oxygen radicals with the application of $1.0 \mathrm{FiO}_{2}$ deserves further study. ${ }^{37,38}$

The decreased survival rate post-ROSC needs to be integrated into parental counseling. During a child's resuscitation, the parents may perceive overwhelming chaos, so having a more informative prognosis during this stressful period could improve the parental expectations. ${ }^{39}$ While emotional support was appreciated by parents who witnessed their child's resuscitation, the most important factor was that they received real-time clinical information from their health care providers. Better expectations could help the parents make more realistic decisions and could even change their attitudes toward end-of-life from life support preferences. ${ }^{40}$

This study reflects the importance of continuous assessment of the progress and impact of changes in CPR practices and outcomes. More accumulation and sharing of reports would aid in the development of better guidelines that are generalizable to all international communities.

\section{Limitations}

Studies on pediatric resuscitation are difficult to perform and frequently include small numbers of patients. ${ }^{29}$ They are mostly retrospective and based on registries or administrative data, rather than being prospective and well-designed studies, so they are vulnerable to the challenges of missing data and potential bias. ${ }^{11}$ However, the pediatric CPR is relatively a rare event and the accumulation of more reports, despite aforementioned limitation, is important for further progress in pediatrics CPR studies. The results of our study should be interpreted with caution due to the limitations of the study, including its retrospective and single-center design. The multivariate analysis in our study revealed interesting findings; however, the fairly large number of comparisons relative to the sample size needs to be interpreted with precaution. The assessment of CPR quality components was difficult to retrieve retrospectively and was beyond the study scope, but it warrants validation in future research. Our PICU adheres to the AHA Guidelines for CPR for pediatric advanced life support (PALS), and it undergoes ongoing revisions to target key questions related to pediatric resuscitation. The PALS Guidelines, which were revised by the AHA in 2015, were published in the "American Heart Association Guidelines Update for Cardiopulmonary Resuscitation and Emergency Cardiovascular Care." These updates are usually applied in real-life settings within a year of their announcement and may affect CPR studies that overlap with these updates. ${ }^{1}$ We also believe that additional prospective data regarding the neurological outcomes of surviving patients could have added more insights to the outcome. However, the neurological outcome was not considered in the study scope. Nevertheless, this study considered relevant and meaningful primary outcomes, with potential expansion to include neurological outcomes in future updates. Although the duration of a CPR event could be difficult to validate in the context of an emergency event, the documentation of CPR in a specifically designed form was quite helpful for tracking, in combination with monitoring the timeline print attached to the form.

\section{Conclusion}

In the PICU setting, better CPR outcomes were associated with younger age, a respiratory cause of arrest, a bradycardia rhythm, and a shorter CPR duration. The duration of CPR remains one of the crucial factors for CPR outcomes and needs to be considered in parallel with the guidelines emphasizing CPR quality. The decreased survival rate post-ROSC needs to be carefully considered during parental counseling, while better anticipation and prevention of CPR remain ongoing challenges. A multicenter national registry of CPR outcomes would further improve and widen the research scope.

\section{Funding \\ None.}

\section{Conflict of Interest}

None declared.

\section{Acknowledgments}

The authors thank the Deanship of Scientific Research and Researchers Support and Services Unit at King Saud University for their technical support. 


\section{References}

1 Girotra S, Spertus JA, Li Y, Berg RA, Nadkarni VM, Chan PSAmerican Heart Association Get With the Guidelines-Resuscitation Investigators. Survival trends in pediatric in-hospital cardiac arrests: an analysis from get with the guidelines-resuscitation. Circ Cardiovasc Qual Outcomes 2013;6(01):42-49

2 Alten JA, Klugman D, Raymond TT, et al. Epidemiology and outcomes of cardiac arrest in pediatric cardiac ICUs. Pediatr Crit Care Med 2017;18(10):935-943

3 Berg RA, Sutton RM, Holubkov R, et al; Eunice Kennedy Shriver National Institute of Child Health and Human Development Collaborative Pediatric Critical Care Research Network and for the American Heart Association's Get With the Guidelines-Resuscitation (formerly the National Registry of Cardiopulmonary Resuscitation) Investigators. Ratio of PICU versus ward cardiopulmonary resuscitation events is increasing. Crit Care Med 2013;41 (10):2292-2297

4 Scholz SS, Borgstedt R, Menzel LC, Rehberg S, Jansen G. Evolution and current state of global research on paediatric resuscitation: a systematic scientometric analysis. Scand J Trauma Resusc Emerg Med 2020;28(01):90

5 de Caen AR, Berg MD, Chameides L, et al. Part 12: pediatric advanced life support: 2015 American Heart Association Guidelines Update for Cardiopulmonary Resuscitation and Emergency Cardiovascular Care (reprint). Pediatrics 2015;136(Suppl 2): S176-S195 F

6 Morgan RW, Landis WP, Marquez A, et al. Hemodynamic effects of chest compression interruptions during pediatric in-hospital cardiopulmonary resuscitation. Resuscitation 2019;139:1-8

7 Topjian AA, Nadkarni VM, Berg RA. Cardiopulmonary resuscitation in children. Curr Opin Crit Care 2009;15(03):203-208

8 Nasser BA, Idris J, Mesned AR, Mohamad T, Kabbani MS, Alakfash A. Predictors of cardio pulmonary resuscitation outcome in postoperative cardiac children. J Saudi Heart Assoc 2016;28 (04):244-248

9 Kaki AM, Alghalayini KW, Alama MN, et al. An audit of in-hospital cardiopulmonary resuscitation in a teaching hospital in Saudi Arabia: A retrospective study. Saudi J Anaesth 2017;11(04):415-420

10 Young KD, Seidel JS. Pediatric cardiopulmonary resuscitation: a collective review. Ann Emerg Med 1999;33(02):195-205

11 Berg RA, Nadkarni VM, Clark AE, et al; Eunice Kennedy Shriver National Institute of Child Health and Human Development Collaborative Pediatric Critical Care Research Network. Incidence and outcomes of cardiopulmonary resuscitation in PICUs. Crit Care Med 2016;44(04):798-808

12 Matos RI, Watson RS, Nadkarni VM, et al; American Heart Association's Get With The Guidelines-Resuscitation (Formerly the National Registry of Cardiopulmonary Resuscitation) Investigators. Duration of cardiopulmonary resuscitation and illness category impact survival and neurologic outcomes for in-hospital pediatric cardiac arrests. Circulation 2013;127(04):442-451

13 Bhanji F, Topjian AA, Nadkarni VM, et al; American Heart Association's Get With the Guidelines-Resuscitation Investigators. Survival rates following pediatric in-hospital cardiac arrests during nights and weekends. JAMA Pediatr 2017;171(01):39-45

14 Parra DA, Totapally BR, Zahn E, et al. Outcome of cardiopulmonary resuscitation in a pediatric cardiac intensive care unit. Crit Care Med 2000;28(09):3296-3300

15 Wu E-T, Li M-J, Huang S-C, et al. Survey of outcome of CPR in pediatric in-hospital cardiac arrest in a medical center in Taiwan. Resuscitation 2009;80(04):443-448

16 Genbrugge C, Eertmans W, Salcido DD. Monitor the quality of cardiopulmonary resuscitation in 2020. Curr Opin Crit Care 2020; 26(03):219-227

17 Hunt EA, Jeffers J, McNamara L, et al. Improved cardiopulmonary resuscitation performance with CODE ACES2: a resuscitation quality bundle. J Am Heart Assoc 2018;7(24):e009860
18 Niles DE, Duval-Arnould J, Skellett S, et al; pediatric Resuscitation Quality (pediRES-Q) Collaborative Investigators. Characterization of pediatric in-hospital cardiopulmonary resuscitation quality metrics across an international resuscitation collaborative. Pediatr Crit Care Med 2018;19(05):421-432

19 Topjian AA, Raymond TT, Atkins D, et al; Pediatric Basic and Advanced Life Support Collaborators. Part 4: Pediatric Basic and Advanced Life Support: 2020 American Heart Association Guidelines for Cardiopulmonary Resuscitation and Emergency Cardiovascular Care. Circulation 2020;142(16_suppl_2):S469-S523

20 Martinez PA, Totapally BR. The epidemiology and outcomes of pediatric in-hospital cardiopulmonary arrest in the United States during 1997 to 2012. Resuscitation 2016;105:177-181

21 Mustafa K, Buckley H, Feltbower R, Kumar R, Scholefield BR. Epidemiology of cardiopulmonary resuscitation in critically ill children admitted to pediatric intensive care units across England: a multicenter retrospective cohort study. J Am Heart Assoc 2021;10(09):e018177

22 Tham LP, Wah W, Phillips R, et al. Epidemiology and outcome of paediatric out-of-hospital cardiac arrests: A paediatric sub-study of the Pan-Asian resuscitation outcomes study (PAROS). Resuscitation 2018;125:111-117

23 Tijssen JA, Prince DK, Morrison LJ, et al; Resuscitation Outcomes Consortium. Time on the scene and interventions are associated with improved survival in pediatric out-of-hospital cardiac arrest. Resuscitation 2015;94:1-7

24 Rajan S, Wissenberg M, Folke F, et al. Out-of-hospital cardiac arrests in children and adolescents: incidences, outcomes, and household socioeconomic status. Resuscitation 2015;88:12-19

25 Berens RJ, Cassidy LD, Matchey J, et al. Probability of survival based on etiology of cardiopulmonary arrest in pediatric patients. Paediatr Anaesth 2011;21(08):834-840

26 Andersen LW, Kurth T, Chase M, et al; American Heart Association's Get With The Guidelines-Resuscitation Investigators. Early administration of epinephrine (adrenaline) in patients with cardiac arrest with initial shockable rhythm in hospital: propensity score matched analysis. BMJ 2016;353:i1577-i1577

27 Mavroudis CD, Ko TS, Morgan RW, et al. Epinephrine's effects on cerebrovascular and systemic hemodynamics during cardiopulmonary resuscitation. Crit Care 2020;24(01):583

28 Hunt EA, Duval-Arnould JM, Bembea MM, et al; American Heart Association's Get With The Guidelines-Resuscitation Investigators. Association between time to defibrillation and survival in pediatric in-hospital cardiac arrest with a first documented shockable rhythm. JAMA Netw Open 2018;1(05):e182643-e182643

29 Jansen G, Borgstedt R, Irmscher L, et al. Incidence, mortality, and characteristics of 18 pediatric perioperative cardiac arrests: an observational trial from 22,650 pediatric anesthesias in a German tertiary care hospital. Anesthes Analges 2021;10:1213

30 Appiah J, Salie S, Argent A, et al. Characteristics, course and outcomes of children admitted to a paediatric intensive care unit after cardiac arrest. South Afr J Crit Care 2018;34:58-64

31 Assar S, Husseinzadeh M, Nikravesh AH, Davoodzadeh H. The success rate of pediatric in-hospital cardiopulmonary resuscitation in Ahvaz training hospitals. Scientifica (Cairo) 2016; 2016:9648140-9648140

32 Esangbedo I, Yu P, Raymond T, et al; Pediatric Resuscitation Quality (pediRES-Q) Collaborative Investigators. Pediatric in-hospital CPR quality at night and on weekends. Resuscitation 2020; 146:56-63

33 Berg RA, Sutton RM, Reeder RW, et al; Eunice Kennedy Shriver National Institute of Child Health and Human Development Collaborative Pediatric Critical Care Research Network (CPCCRN) PICqCPR (Pediatric Intensive Care Quality of Cardio-Pulmonary Resuscitation) Investigators. Association between diastolic blood pressure during pediatric in-hospital cardiopulmonary resuscitation and survival. Circulation 2018;137(17):1784-1795 
34 Wolfe HA, Morgan RW, Sutton RM, et al; Eunice Kennedy Shriver National Institute of Child Health Human Development Collaborative Pediatric Critical Care Research Network Pediatric Intensive Care Quality of Cardiopulmonary Resuscitation (PICqCPR) investigators. Association between time of day and CPR quality as measured by CPR hemodynamics during pediatric in-hospital CPR. Resuscitation 2020;153:209-216

35 Wolfe HA, Sutton RM, Reeder RW, et al; Eunice Kennedy Shriver National Institute of Child Health Human Development Collaborative Pediatric Critical Care Research Network Pediatric Intensive Care Quality of Cardiopulmonary Resuscitation Investigators. Functional outcomes among survivors of pediatric in-hospital cardiac arrest are associated with baseline neurologic and functional status, but not with diastolic blood pressure during CPR. Resuscitation 2019;143:57-65

36 Lasa JJ, Rogers RS, Localio R, et al. Extracorporeal cardiopulmonary resuscitation (e-CPR) during pediatric in-hospital cardiopulmonary arrest is associated with improved survival to discharge: a report from the American Heart Association's Get With The GuidelinesResuscitation (GWTG-R) Regi. Circulation 2016;133(02):165-176

37 Marquez AM, Morgan RW, Ko T, et al. Oxygen exposure during cardiopulmonary resuscitation is associated with cerebral oxidative injury in a randomized, blinded, controlled, preclinical trial. J Am Heart Assoc 2020;9(09):e015032

38 Nelskylä A, Nurmi J, Jousi M, et al. The effect of $50 \%$ compared to $100 \%$ inspired oxygen fraction on brain oxygenation and post cardiac arrest mitochondrial function in experimental cardiac arrest. Resuscitation 2017;116:1-7

39 Stewart SA. Parents' experience during a child's resuscitation: getting through it. J Pediatr Nurs 2019;47:58-67

40 Temsah $\mathrm{M}-\mathrm{H}$. Ethical considerations about changing parental attitude towards end-of-life care in twins with lethal disease. Sudan J Paediatr 2018;18(01):76-82 